\title{
Targeting of Acyl-CoA synthetase 5 decreases jejunal fatty acid activation with no effect on dietary long-chain fatty acid absorption
}

\author{
Nahum Meller, Michelle E Morgan, Winifred PS Wong, Jessica B Altemus and Ephraim Sehayek*
}

\begin{abstract}
Background: The absorption of dietary long chain fatty acids (LCFA) largely occurs in the jejunum. LCFA are activated via conjugation with Coenzyme A (CoA), a reaction catalyzed by Acyl-CoA synthetases (ACS). Acyl-CoA sythesis is critical for dietary LCFA absorption; yet, the jejunal ACS enzymes that catalyze the reaction are largely unknown.

Findings: High throughput mRNA sequencing of the mouse jejunum revealed that the expression of acyl-CoA synthetase 5 (Acs|5) and fatty-acid transport protein 4 (Fatp4) largely exceeded all other annotated ACS genes that activate LCFA. Interestingly, Acsl5 knockout (KO) mice displayed a decrease of $60 \%$ in jejunal total long chain acyl-CoA synthesis rate. Nevertheless, and despite of this decrease, dietary LCFA absorption and body-weight gain in response to high fat diet remained unaffected.

Conclusion: Acsl5 is a major activator of dietary LCFA, yet in AcsI5 KO mice residual ACS activity is sufficient for maintaining a normal LCFA absorption. Our findings provide further evidence for a robust small intestine LCFA absorption capacity.
\end{abstract}

Keywords: Small intestine, Fat absorption, Acsl5, Fatp4, LCFA, Acyl-CoA

\section{Findings}

\section{Background}

Intestinal absorption of dietary long chain fatty acids (LCFA) is an important determinant of body weight and obesity-related metabolic disorders. LCFA absorption largely occurs in the jejunum where these nutrients undergo uptake across the brush border membrane of enterocytes. LCFA uptake is followed by a critical activation step through conjugation with coenzyme-A (CoA). In enterocytes, dietary LCFA-CoA conjugates are primarily utilized for triglycerides (TG) synthesis, packed into chylomicrons and secreted through the basolateral membrane [1,2]. LCFA conjugation with $\mathrm{CoA}$ is catalyzed by Acyl-CoA synthetases (ACS). Mammals possess 13 annotated ACS genes that activate LCFA and clustered in three different gene families: ACSL (acyl-CoA synthetase long chain),

\footnotetext{
* Correspondence: sehayee@ccf.org

Ephraim Sehayek, Genomic Medicine Institute, The Cleveland Clinic Foundation, 9500 Euclid Ave, Cleveland OH 44195, USA
}

ACSBG (acyl-CoA synthetase bubblegum) and FATP (fatty acid transport proteins; also designated ACSVL or SLC27) [3-5]. The impact of each of these genes on intestinal absorption of LCFA is largely unknown.

\section{Methods}

The methods used are described briefly at the figure legends. Detailed description of mice, diets and assays used in the study is presented in Additional files 1 and 2. All experiments involving mice were approved by the Cleveland Clinic Animal Care and Use Committee.

\section{Results}

Expression of long chain acyl-CoA synthetase genes in C57BL/6J jejunum was determined by transcriptome shotgun sequencing analysis (mRNA-seq). As shown in Figure 1, out of the 13 annotated LCFA acyl-CoA synthetase genes, the number of properly paired reads that were aligned to Acsl5 and Fatp4 largely exceeded the number of reads that were aligned to the other genes. A previous study has shown that Fatp4 is 


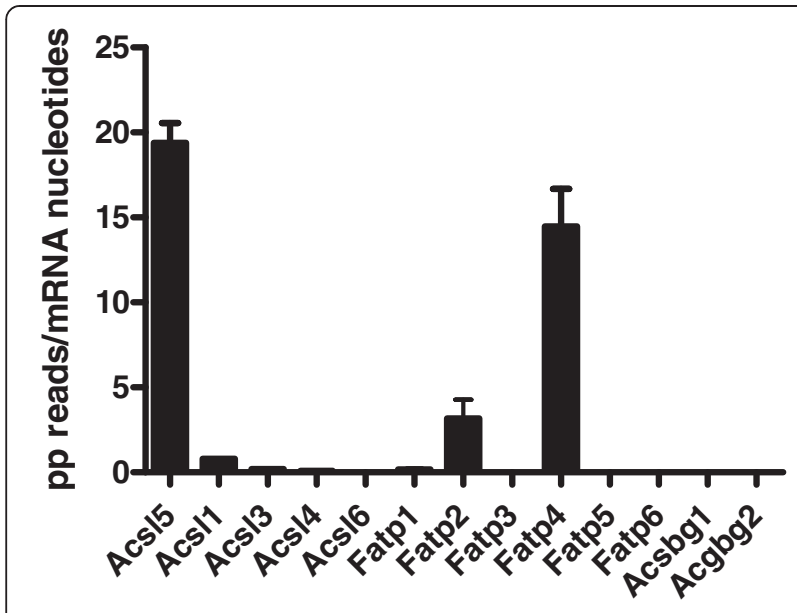

Figure 1 AcsI5 and Fatp4 are the two predominant LCFA CoA-synthetases in mouse jejunum. Five C57BL/6J females were fasted for 6 hours, sacrificed, the jejunum harvested, polyadenylated RNA isolated, CDNA synthesized and sequenced. Sequencing reads were aligned against a reference mouse genome. Gene expression levels were calculated as mean \pm SD of properly paired (PP)-end reads aligned to each gene after normalization for mRNA length in basepairs. Expression levels of the 13 annotated ACS genes is presented. dispensable for dietary fat absorption [6], therefore, we focused our efforts on elucidating the role of Acsl5. Quantitative PCR and Western blotting demonstrated that Acsl5 is preferentially expressed in the jejunum (Additional file 3). These results suggested a role for Acsl5 in determining the jejunal absorption of LCFA.

To determine the role of Acsl5 in jejunal LCFA-CoA synthesis and absorption, we have used mice that were targeted for this gene (characterization of targeted animals is found in Additional file 2). Jejunum mucosal homogenates were assayed for acyl-CoA synthesis rate by using palmitic acid, a prototype member of the long chain fatty acid family, as a substrate. As shown in Figure 2A, as compared to wild-type littermate controls, Acsl5-KO males and females displayed a decrease of $60 \%$ in jejunal palmitoyl-CoA synthesis rate. Animals heterozygous for the targeted allele displayed only a moderate $15-35 \%$ decrease in activity, which reached statistical significance only in females. When compared to the jejunum, liver Acsl5 expression is diminished (Additional file 3). Consistent with these findings, liver synthesis rates of palmitoyl-CoA in Acsl5 $\mathrm{KO}$ mice were fully conserved (Figure $2 \mathrm{~B}$ ).

To determine the effect of Acsl5 targeting on LCFA absorption, mice were fasted overnight, lipases activity inhibited through intravenous injection of tyloxapol, animals provided with a gastric bolus of olive oil supplemented with $\left[{ }^{3} \mathrm{H}\right]$-radiolabeled oleic acid, and levels of
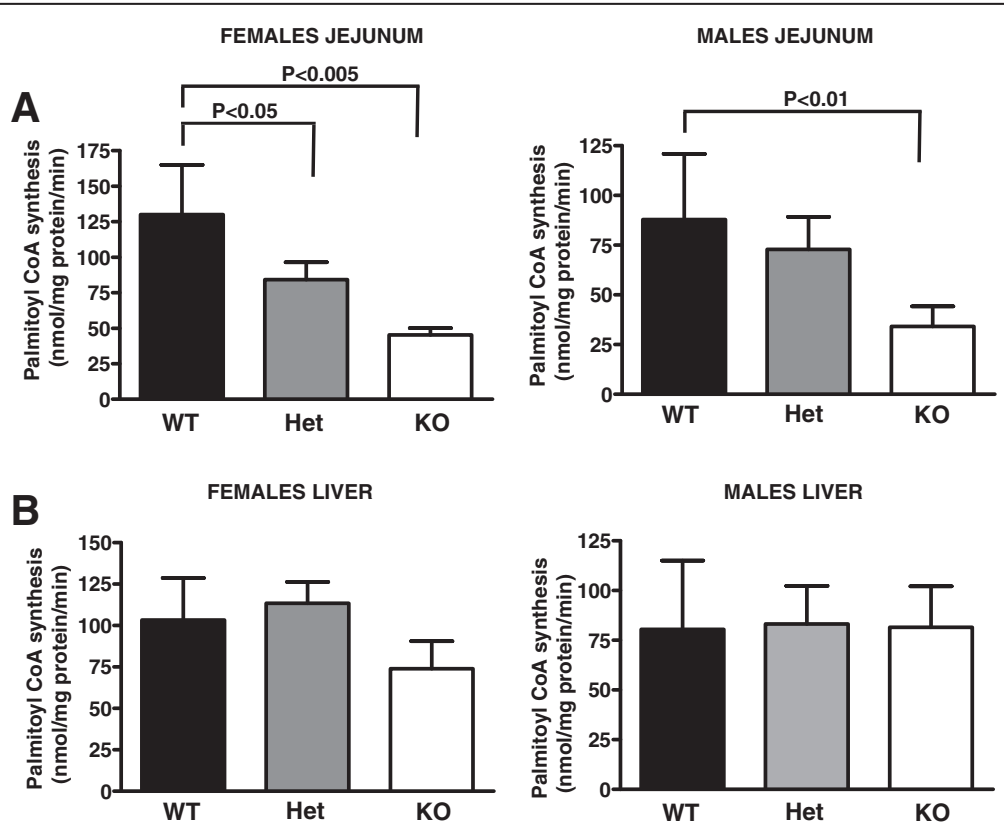

Figure 2 Reduced total jejunal ACS activity in Acsl5 KO mice. Jejunal mucosa (A) and liver tissues (B) from female or male Acsl5 wild type (WT), heterozygous (Het) or knockout (KO) mice, 5 animals per group, were harvested and homogenized. Equal amounts of protein were incubated in the presence of raiolabeled palmitic acid, ATP and CoA. Unconjugated fatty acid was extracted in heptane and aqueous phase radioactivity, containing the water soluble palmitoyl-CoA was determined by scintillation counting. Mean \pm SD palmitoyl-CoA synthesis rates are presented. 

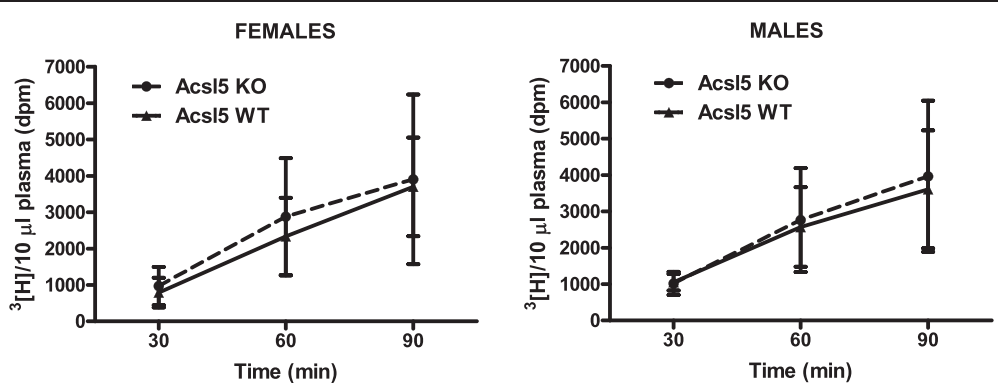

Figure 3 AcsI5 KO mice display a normal dietary LCFA absorption from the intestine. AcsI5 WT and KO mice, females or males, ( 5 per group), were fasted overnight and than intravenously injected with tyloxapol to inhibit vascular lipases. Thirty minutes later the mice received a gastric bolus olive oil supplemented with radiolabeled oleic acid and retroorbital sinus blood samples were collected at 30, 60 and 90 min post gavage. Plasma radioactivity was determined by scintillation counting.

the radioactive tracer were measured in plasma samples collected for the first 90 minutes post gastric gavage. As shown in Figure 3, as compared to littermate wild-type controls, Acsl5-KO males and females displayed undistinguishable plasma accumulation of the radiolabeled tracer. These finding suggested that targeting of the Acsl5 gene has no impact on intestinal LCFA absorption.

To determine the effect of Acsl5 targeting on bodyweight gain in response to high fat diet, mice were fed a Western-type diet for 13 weeks. As shown in Figure 4, Acsl5-KO males and females displayed weight gain that was indistinguishable of their littermate wild-type controls. Finally, targeting of Acsl5 had no effect on dietary cholesterol absorption, plasma cholesterol levels, plasma triglycerides or fasting plasma glucose levels (data not shown).

\section{Discussion}

We identified Acsl5 as a major ACS gene expressed in the jejunum (Figure 1). Interestingly, while Acsl5 targeting resulted in a substantial decrease in jejunal total LCFA ACS activity (Figure 2) this decrease was not sufficient to reduce either LCFA absorption or body weight gain in response to high fat diet (Figures 3 and 4). As expression levels of other ACS genes in the jejunum remained stable
(Additional file 2), these findings are consistent with a large capacity of the small intestine to absorb dietary LCFA. Therefore, even a reduction of $60 \%$ in total ACS activity is insufficient in affecting the absorption of LCFA even under conditions where the absorption process is stressed by the feeding of a high fat diet.

Our mRNA-Seq results suggest that jejunum Acsl5 and Fatp4 are expressed at a comparable level and together account for over $90 \%$ of the LCFA ACS transcripts in this tissue (Figure 1). A previous study reported, through targeting of Fatp4, that this enzyme is dispensable for intestinal fat absorption [6]. Unfortunately the authors have not reported the effect of Fatp4 targeting on jejunal LCFA-CoA synthesis rate. Interestingly, studies in newly born Fatp4-KO mice demonstrated $~ 10$ fold inhibition in activation of the very long FA lignoceric acid (C24:0) but no inhibition of the LCFA palmitic or oleic acids [7]. These findings may suggest that Fatp4 is not a major determinant of LCFA absorption from the intestine. Nevertheless, studies in Acsl5/intestinal-Fatp4 double $\mathrm{KO}$, that are beyond the scope of this report, are needed to clarify the combined effect of Acsl5 and Fatp4. It is of note that Fatp2, amongst the three highly-expressed LCFA ACS gene in the jejunum (Figure 1), may also contribute to LCFA absorption
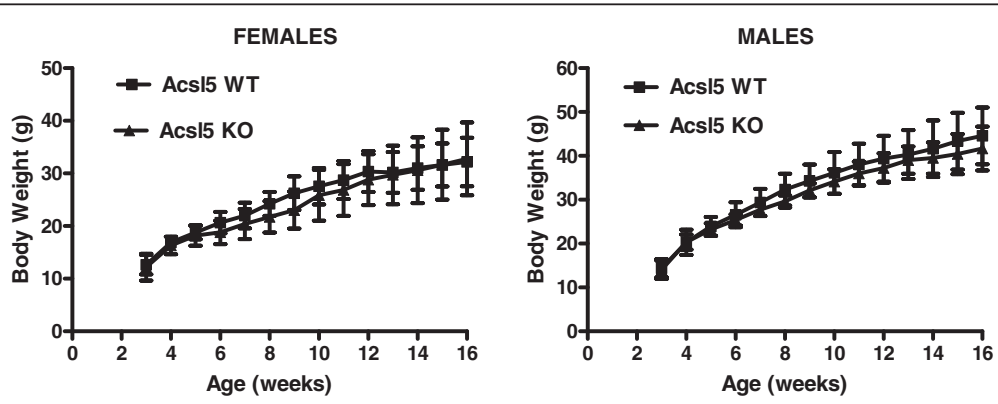

Figure 4 AcsI5 KO display normal weight gain in response to high fat diet. Acsl5 WT and KO mice, females or males, (5 per group), were fed a Western type diet for 13 weeks and body weights were recorded. No significant differences are observed between Acsl5 KO and WT animals. 
and the lack of phonotype in our Acsl5 $\mathrm{KO}$ mice. Finally, we cannot exclude the possibility that additional, not as yet identified, ACS genes may contribute to absence of phenotype in Acsl5 knockouts.

\section{Additional files}

Additional file 1: Methods. Jejunum mRNA sequencing and analysis. Quantitative PCR. Western blotting Analysis. Total acyl-CoA synthesis assay. Dietary LCFA absorption assay. Statistical analysis.

Additional file 2: AcsI5 KO mice. Animals and diets description. Acsl5 protein expression in WT, Heterozygote and KO mice. qPCR analysis of expression of major ACS genes and genes involved in fat absorption in Acsl5 $\mathrm{KO}$ and WT mice jejunum.

Additional file 3: Acsl5 is preferentially expressed in the jejunum. RNA and protein levels of Acsl1 and Acsl5 in tissues with active fatty acid metabolism and along the longitudinal axis of the gastrointestinal tract.

\section{Abbreviations}

CoA: Coenzyme A; ACS: Acyl-CoA synthetase; LCFA: Long chain fatty acids; TG: Triglycerides; KO: Knockout; Acsl: Acyl-CoA synthetases long chain; Fatp: Fatty acid transport protein.

\section{Competing interests}

The authors declare that they have no competing interests to report.

\section{Authors' contributions}

ES and NM designed the research and drafted the manuscript; MM, WW, JA and NM performed the experiments and analyzed the results. All authors read and approved the final manuscript.

\section{Acknowledgments}

We thank Dr. David Serre at The Cleveland Clinic for his excellent support in analyzing the mRNA-Seq data. We thank Dr. Julia Meller for her help in preparing the manuscript for publication. These studies were supported by funding from NHLBI (HL092991 to ES).

Received: 12 April 2013 Accepted: 10 June 2013

Published: 14 June 2013

\section{References}

1. Kindel T, Lee DM, Tso P: The mechanism of the formation and secretion of chylomicrons. Atheroscler Suppl 2010, 11:11-16.

2. Iqbal J, Hussain MM: Intestinal lipid absorption. Am J Physiol Endocrinol Metab 2009, 296:E1183-E1194.

3. Watkins PA, Maiquel D, Jia Z, Pevsner J: Evidence for 26 distinct acyl-coenzyme A synthetase genes in the human genome. J Lipid Res 2007, 48:2736-2750

4. Watkins PA, Ellis JM: Peroxisomal acyl-CoA synthetases. Biochim Biophys Acta 1822, 2012:1411-1420.

5. Mashek DG, Li LO, Coleman RA: Long-chain acyl-CoA synthetases and fatty acid channeling. Future Lipidol 2007, 2:465-476.

6. Shim J, Moulson CL, Newberry EP, Lin MH, Xie Y, Kennedy SM, Miner JH, Davidson NO: Fatty acid transport protein 4 is dispensable for intestinal lipid absorption in mice. J Lipid Res 2009, 50:491-500.

7. Hall AM, Wiczer BM, Herrmann T, Stremmel W, Bernlohr DA: Enzymatic properties of purified murine fatty acid transport protein 4 and analysis of acyl-CoA synthetase activities in tissues from FATP4 null mice. J Biol Chem 2005, 280:11948-11954.

doi:10.1186/1476-511X-12-88

Cite this article as: Meller et al: Targeting of Acyl-CoA synthetase 5 decreases jejunal fatty acid activation with no effect on dietary longchain fatty acid absorption. Lipids in Health and Disease 2013 12:88.

\section{Submit your next manuscript to BioMed Central and take full advantage of:}

- Convenient online submission

- Thorough peer review

- No space constraints or color figure charges

- Immediate publication on acceptance

- Inclusion in PubMed, CAS, Scopus and Google Scholar

- Research which is freely available for redistribution 